\title{
The Influence of Levetiracetam in Cognitive Performance in Healthy Individuals: Neuropsychological, Behavioral and Electrophysiological Approach
}

\author{
Julio Cesar Magalhães ${ }^{1}$, Mariana Gongora ${ }^{1}$, Renan Vicente ${ }^{1,2}$, Juliana Bittencourt ${ }^{1,4,7}$, Guaraci Tanaka ${ }^{1}$, Bruna \\ Velasques $^{1,2,4}$, Silmar Teixeira, Gledys Morato, ${ }^{5}$, Luis F. Basile ${ }^{6}$, Oscar Arias-Carrión ${ }^{8,9}$, Fernando A.M.S Pompeu, \\ Mauricio Cagy ${ }^{3}$, Pedro Ribeiro ${ }^{1,2,4}$ \\ 'Brain Mapping and Sensory Motor Integration, Institute of Psychiatry, Federal University of Rio de Janeiro (IPUB/UFRJ), Rio de Janeiro, \\ ${ }^{2}$ Bioscience Department (EEFD/UFRJ), School of Physical Education, ${ }^{3}$ Biomedical Engineering Program, COPPE, Federal University of Rio \\ de Janeiro, Rio de Janeiro, ${ }^{4}$ Institute of Applied Neuroscience (INA), Rio de Janeiro, ${ }^{5}$ Brain Mapping and Plasticity Laboratory, Federal \\ University of Piauí, Teresina, ${ }^{6}$ Laboratory of Psychophysiology, Faculdade da Saúde, UMESP, São Paulo, ${ }^{7}$ Veiga de Almeida University, \\ Rio de Janeiro, Brazil, ${ }^{8}$ Movement Disorders and Transcranial Magnetic Stimulation Unit, Hospital General Dr. Manuel Gea González, Mexico \\ D.F., ${ }^{9}$ Neurology Department, Hospital General Ajusco Medio, Mexico D.F., Mexico
}

Objective: The present study sought to analyze the influence of Levetiracetam (LEV) in cognitive performance by identifying the changes produced by LEV in reaction time, in neuropsychological assessment of attention and memory and in absolute theta power in frontal activity.

Methods: Twelve healthy subjects ( 5 men and 7 women; mean age, 30.08 years, standard deviation, 4.71) were recruited for this study. The neuropsychological tests: Trail Making Test (A and B), Digit Span (direct and indirect numerical orders/working memory); Stroop test (inhibitory control of attention); Tower of London (planning and decision-making) and a quantitative electroencephalography were applied in 2 different days after and before the participants ingested the capsule of placebo or 500 mg LEV.

Results: A two-way-ANOVA was implemented to observe the interaction between conditions (placebo or LEV $500 \mathrm{mg}$ ) and moments (pre- and post-ingestion of LEV or placebo). The data were analyzed by the SPSS statistical package $(p<0.05)$. For the neuropsychological parameter, the Trail Making Test $(A)$ was the only test that showed significant difference for condition in the task execution time $(p=0.026)$. Regarding the reaction time in the behavioral parameter, an interaction between both factors $(p=0.034)$ was identified through a two-way-ANOVA (condition versus moment). Electrophysiological measures showed a significant interaction for electrodes: F7, F3, and FZ.

Conclusion: The findings showed that LEV promotes an important cognitive enhancement in the executive functions.

KEY WORDS: Levetiracetam; Neuropsychological tests; Reaction time; Electroencephalography; Absolute theta,

\section{INTRODUCTION}

The improvement of cognitive and perceptual functions is considered to be one of the frontiers of knowledge. ${ }^{1)}$ The possibility of expanding our capacities of attention, alertness, memory, perception, information integration, decision making, planning, and cognitive flexibility is a long-term goal for neuroscience. ${ }^{2)}$ Cognitive enhancers

\footnotetext{
Received: October 14, 2014 / Revised: December 2, 2014

Accepted: December 23, 2014

Address for correspondence: Mariana Gongora, PhD Brain Mapping and Sensory Motor Integration, Institute of Psychiatry, Federal University of Rio de Janeiro (IPUB/UFRJ), Rua Cel. Moreira Cesar, 375 apto. 802. Icaraí- Niterói, RJ. Cep 24230-054, Brazil

Tel: +55-21-2711-2530, Fax: +55-21-2259-8129

E-mail: marianagongora@gmail.com
}

were actually born from several attempts to assist cognitive functions in full decline due to specific diseases, such as dementia, attention deficit disorder and other various psychiatric disorders. ${ }^{3-5)}$ In an attempt to achieve a specific solution for certain diseases, the possibility of a significant change in brain efficiency was envisioned through the use of drugs that could help to restore or improve specific cognitive functions. Since then, several drugs have been developed in order to improve or expand cognitive aspects. ${ }^{6}$

Among cognitive enhancers, we can find psychostimulants (methylphenidate and amphetamines), wakefulness promoters (modafinil) and glutamate activators (ampakines). Considering this, methylphenidate is a strong inhibitor of dopamine and noradrenaline reuptake. In turn,

(a) This is an Open-Access article distributed under the terms of the Creative Commons Attribution Non-Commercial License (http://creativecommons.org/licenses/by-nc/3.0) which permits unrestricted non-commercial use, distribution, and reproduction in any medium, provided the original work is properly cited. 
besides inhibiting the dopamine transporter proteins (reuptake), amphetamines also act on the norepinefrine existing in the nerve cells, thus blocking its reabsorption and increasing the concentration of these neurotransmitters in the synaptic cleft. ${ }^{7)}$ Therefore, modafinil also seems to be involved in the reuptake of dopamine transporters, causing a substantial increase in the dopamine extracellular level and synaptic concentration. ${ }^{8)}$ And finally, there are glutamate activators, such as ampakine, which is a positive allosteric modulator of $\alpha$-amino-3-hydroxy-5methyl-4-isoxazolepropionic acid (AMPA) receptors. ${ }^{9,10)}$

In this experiment we used Levetiracetam (LEV) (5enantiomer of 5- $\alpha$-ethyl-2-oxo-1-pyrrolidine acetamide) to test the hypothesis of its effects associated with the improvement of cognition. ${ }^{11)}$ LEV has an extremely positive result in epileptic frames, acting as an anticonvulsant. Unlike cognitive enhancers presented before, LEV engages with the synaptic vesicle glycoprotein (SV2A) and also inhibits the presynaptic calcium channels, reducing the neurotransmitter release, acting as a neuromodulator, thereby preventing the nervous impulse conduction in the synaptic cleft. There are few studies demonstrating the efficacy of LEV in epileptic patients (children and adults), and we know an even smaller number of experiments involving the effects of LEV in healthy individuals. ${ }^{12,13)}$ Due to the sensitivity of the measures, neuropsychological parameters may have great difficulty in identifying the effects of LEV when compared to the placebo condition, especially in healthy individuals.

Therefore, quantitative electroencephalography (qEEG) has been employed as a useful tool to examine possible electrophysiological changes, in particular, the physicochemical aspect of the subject/drug interactions in several experiments. ${ }^{14,15)}$ In addition to the great sensitivity of the measure to detect assumed changes, it is possible to identify aspects of brain function and regulation through the presence or absence of a particular activity (frequency) in the prefrontal and frontal areas of the cerebral cortex using qEEG. These are critical regions for executive functions, and several results demonstrate that theta is involved in a wide range of executive functions, such as sustained attention, working memory, episodic and semantic memory and spatial aspects related to navigation and the ability to imagine objects or actions. ${ }^{16,17)}$ That's why we observed the behavior of the absolute theta power (4 to 8 $\mathrm{Hz}$ ). In an attempt to elucidate the effects of LEV in a population of healthy individuals as a cognitive amplifier, this study aims to examine alleged behavioral changes (reaction time), neuropsychological (attention, working memory, planning and decision making) and electrophysiological (absolute theta power) changes in the frontal region.

\section{METHODS}

\section{Sample}

Twelve healthy subjects (five men and seven women; mean age, 30.08 years, standard deviation [SD], 4.71) were recruited for this study. All subjects were right-handed and had normal or corrected-to-normal vision. Inclusion criteria were absence of mental or physical impairments and no history of psychoactive or psychotropic substance use (as confirmed by anamnesis and clinical examination). A questionnaire was applied to verify if the participants met the inclusion criteria. Subjects were not included if they had slept less than 8 hours prior to the experiment and/or had ingested caffeine within the 48 hours prior to the experiment. All subjects were made aware of the entire experimental protocol and signed a consent form before participating in the study, which was approved by the Ethics Committee at the Federal University of Rio de Janeiro (CAAE:16342213.4.0000.5263) according to the Declaration of Helsinki (1964).

\section{EEG Data Acquisition}

The electroencephalography signal acquisition was recorded using the 20-channel Braintech3000 (EMSA Medical Instruments, Rio de Janeiro, Brazil) EEG system. This program was employed to filter the data: Notch (60 $\mathrm{Hz}$ ), high-pass of $0.3 \mathrm{~Hz}$ and low-pass of $25 \mathrm{~Hz}$ (order 2 Butterworth). Twenty-one electrodes were arranged on a lycra cap (EletroCap Inc., Fairfax, VA, USA) along the scalp on the frontal, temporal, parietal and occipital areas, according to the $10 / 20$ system protocol, ${ }^{18)}$ and two more electrodes were positioned on the earlobes, set as a reference point, yielding 20 mono-pole derivations to them (using Fpz as ground electrode). The caps were individually adjusted and put on each subject, according to each individual's circumference and anatomy proportions. The signal correspondent to each EEG derivation resulted from the electric potential difference between each electrode and the pre-established reference (earlobes). First, the impedance levels of each electrode were calculated, and they were kept below $5 \mathrm{k} \Omega$. The ocular electric activity was estimated by attaching two 9-mm-diameter electrodes in a bipolar montage. The electrodes were positioned, respectively, above and below the right eye orbit, in order to register vertical ocular movements, and on the 
external corner of the same eye, in order to register horizontal ocular movements. Visual artifacts were $a$ priori inspected through a data visualization program using the Matlab 5.3 ${ }^{\circledR}$ (The Mathworks Inc., Natick, MA, USA).

\section{EEG Data Processing}

The electroencephalographic signals collected during the experiment were processed using methods developed by the Brain Mapping and Sensorimotor Integration Laboratory of the Psychiatry Institute of the Federal University of Rio de Janeiro in a Matlab $5.3^{\circledR}$ environment. Visual inspection and independent component analysis (ICA) were applied to quantify reference-free data by removing possible sources of task-induced artifacts. Data from individual electrodes exhibiting loss of contact with the scalp or high impedances $(>5 \mathrm{k} \Omega)$ were deleted as were data from single-trial epochs that exhibited excessive movement artifact $( \pm 100 \mu \mathrm{V})$. ICA was then applied to identify and remove any artifacts that remained after the initial visual inspection. ICA is an information maximization algorithm that derives spatial filters by blind source separation of the EEG signals into temporally independent and spatially fixed components. Independent components resembling an eye blink or muscle artifact were removed, and the remaining components were then projected back onto the scalp electrodes by multiplying the input data by the inverse matrix of the spatial filter coefficients derived from ICA using established procedures. The ICA-filtered data were then re-inspected for residual artifacts using the rejection criteria described above.

\section{Experiment Design}

The experiment was randomized and double-blind, applied on two different days with an interval of at least one week: one day having ingested 1 gelatin capsule with 500 $\mathrm{mg}$ of starch (placebo), and one day having ingested 1 gelatin capsule with $500 \mathrm{mg}$ of LEV. All individuals had to be familiar with the experimental task. The procedures were standardized in the following sequence: application of neuropsychological tests, reaction time, and EEG record at rest. After this, the participants ingested the capsule of placebo or $500 \mathrm{mg}$ LEV. Only after two hours from ingestion, the same previous steps (i.e., application of neuropsychological tests, reaction time and rest EEG) were repeated, characterizing the 'pre' and 'post' intake moments.

\section{Dependent Variables: Neuropsychological Assessments, Behavioral Parameters (Reaction Time) and Electro- encephalographic Measure (Absolute Theta Power)}

The following neuropsychological tests were applied to individuals: Trail Making Test (A and B), Digit Span (direct and indirect numerical orders/working memory), Stroop test (inhibitory control of attention) and the Tower of London (planning and decision-making). This neuropsychological battery has been applied on similar studies that utilized different drugs that also acted on cognition. ${ }^{19-21)}$ Other studies have used similar neuropsychological tests to measure such executive functions. ${ }^{22,23)}$ Software developed in our own laboratory (DELPHI 5.0) was used to analyze the reaction time. Participants had to choose a square (25\% of total shapes) among various circles, identifying the reaction time. In terms of electroencephalographic measure, we used absolute theta power to examine the energy variation among the placebo and LEV conditions.

\section{Cortical Area and EEG Frequency}

We chose to analyze the frontal region, due to its strong relationship with the executive functions. In particular, we focused on processes related with attention, planning and short-term memory. We used the theta frequency range, due to its correlation with attentional processes and working memory. In general, we seek to discuss theta as having a behavior similar to alpha; in other words, the greater presence of theta somehow shows an inhibition of this cortical region. $^{24)}$

\section{Statistical Analysis}

The results were represented as mean and SD. Consequently, a two-way-ANOVA was implemented to observe the interaction between conditions (placebo or $500 \mathrm{mg}$ LEV) and moments (pre- and post-use of LEV or placebo). Additionally, a paired $t$-test was performed to compare the moments (pre vs. post) within each condition aiming to explore the interaction. The significance criterion was $p \leq 0.05$ for all analyses.

To observe two independents factors: condition (LEV and placebo) and moment (before and after drug ingestion). For those electrodes where we found interaction between factors, a paired t-test was applied, in order to examine the possible differences. Each electrode was seen separated, exactly to avoid the Type I error. 


\section{RESULTS}

The present study examined the neuropsychological, behavioral and electrophysiological effects on subjects who ingested the LEV drug. To date, this substance has been used in medicines for epilepsy patients. In this section, we will describe the findings according to three parameters: i) neuropsychological, ii) behavioral, and iii) electrophysiological.

\section{Neuropsychological and Behavioral Parameters}

In order to examine alleged neuropsychological changes due to the use of LEV, a two-way-ANOVA was performed. Specifically, two independent variables were observed: condition (placebo or LEV) and moment (preand post-ingestion of the LEV or placebo). Thus, the re-

Table 1. Results of neuropsychological and behavioral parameters

\begin{tabular}{lc}
\hline \multicolumn{1}{c}{ Parameter } & Mean \pm SD \\
\hline Trail Making Test $(A)^{\star}$ & \\
Execution time statistics & \\
Main effect for condition $(F=5.322 ; p=0.026)$ & $34.0000 \pm 11.97461$ \\
$\quad$ Placebo & $27.7500 \pm 6.29182$ \\
$\quad$ Levetiracetam & \\
Number of errors statistics & \\
Main effect for moment $(F=5.851 ; p=0.020)$ & $0.0000 \pm 0.00000$ \\
Pre-drug ingestion & $0.2083 \pm 0.41485$ \\
Post-drug ingestion & \\
Reaction time ${ }^{\dagger}$ & \\
Main effect for condition $(F=9.292 ; p=0.002)$ & \\
\hline
\end{tabular}

* Mean and standard deviation (SD) of main effect for condition at execution time and main effect for moment at number of statistical errors for Trail Making Test (A).

${ }^{+}$Mean and SD of main effect for condition at reaction time.

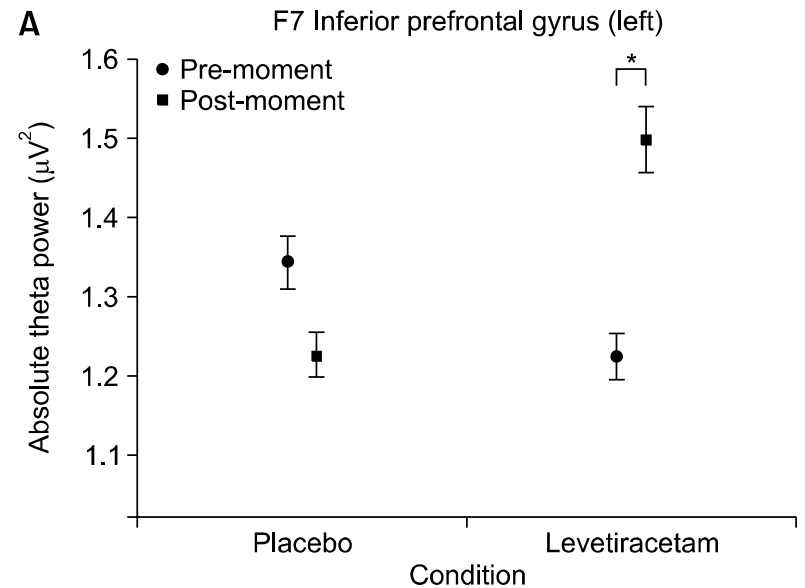

sults did not show any interaction or main effect using the following tests: Trail Making Test (B), Digit Span, Stroop test and Tower of London. In particular, the Trail Making Test (A) was the only test that showed significant difference. A main effect for condition was found at the task execution time $(\mathrm{F}=5.322 ; p=0.026)$, and a main effect for moment was found in the number of errors committed during the test $(\mathrm{F}=5.851 ; p=0.020)$. The reaction time was also analyzed by a two-way-ANOVA (condition vs. moment $)$, and a main effect for condition $(\mathrm{F}=9.292 ; p=0.002)$ was found (Table 1).

\section{Electrophysiological Parameter (qEEG)}

First, the interaction (condition versus moment) results are presented, followed by the main effects. The following electrodes were analyzed: FP1, FP2, F3, FZ, F4, F7 and F8. A significant interaction was observed for the following electrodes: $\mathrm{F} 7(\mathrm{~F}=33.360, p=0.001$; Fig. $1 \mathrm{~A})$ and $\mathrm{F} 3$ $(\mathrm{F}=5.475, p=0.019$; Fig. $2 \mathrm{~A})$. A main effect for condition was also found for the following electrodes: FP1 $(\mathrm{F}=4.246, p=0.039$; Fig. $3 \mathrm{~A}), \mathrm{FP} 2(\mathrm{~F}=3.909, p=0.048$; Fig. 3B), $\mathrm{F} 8$ ( $\mathrm{F}=4.753, p=0.029$; Fig. $1 \mathrm{~B}), \mathrm{Fz}(\mathrm{F}=4.913$, $p=0.027)$ and $\mathrm{F} 4(\mathrm{~F}=15.269, p=0.000$; Fig. $2 \mathrm{C})$. Finally, significant changes for the main effect for moment were shown for the following electrodes: FP2 $(\mathrm{F}=10.317$, $p=0.001$; Fig. $3 \mathrm{C})$ and $\mathrm{F} 4(\mathrm{~F}=6.482, p=0.011$; Fig. 2D).

\section{DISCUSSION}

The present study aimed to investigate the behavioral, neuropsychological and electrophysiological changes produced by LEV in an attempt to explore its effects as a

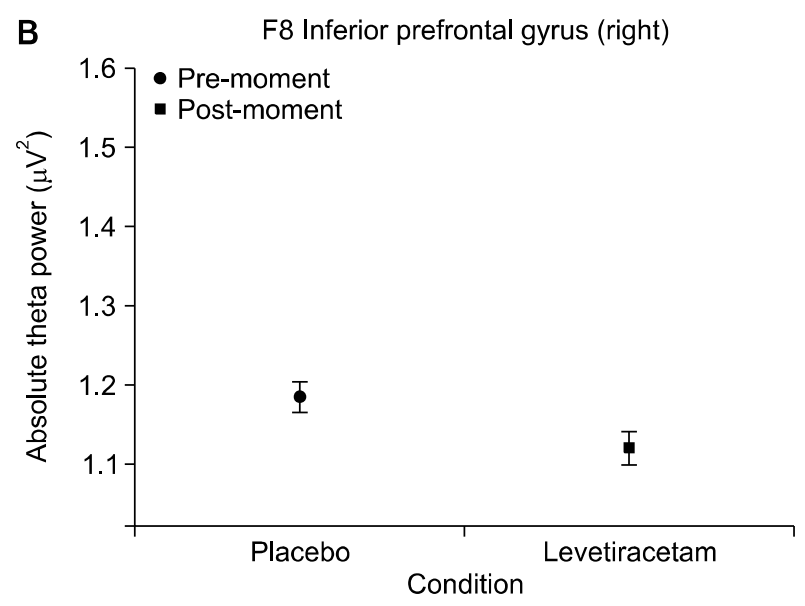

Fig. 1. Mean and standard error of absolute theta power. (A) Left inferior prefrontal gyrus (F7) interaction between conditions (placebo and levetiracetam) and moments (pre and post) $(p=0.001)$. The paired $t$-test for post moment demonstrated significant difference between conditions ( $p=0.004)$; $(B)$ right prefrontal gyrus (F8) on the placebo and levetiracetam conditions $(p=0.029)$. 

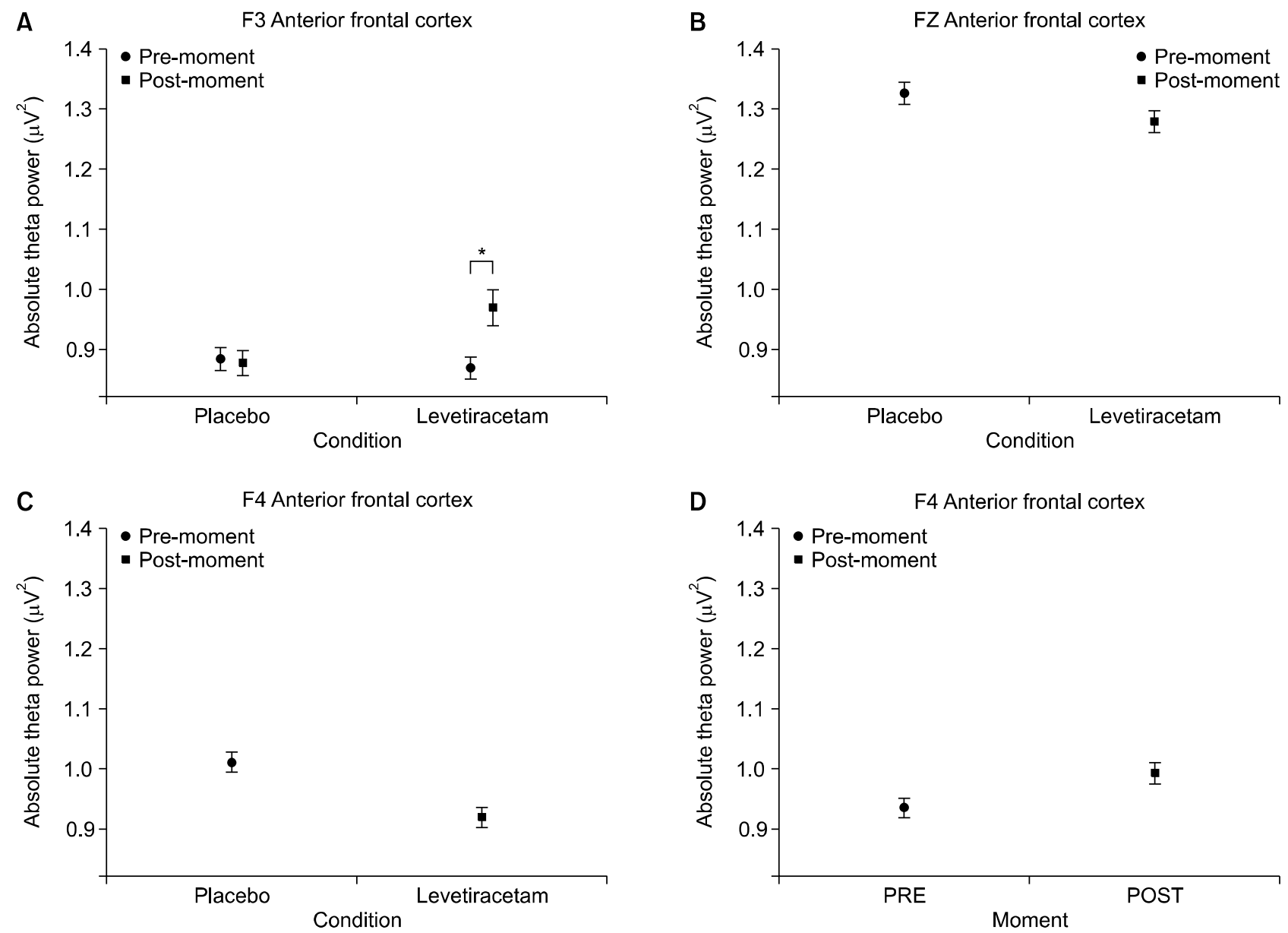

Fig. 2. Mean and standard error of absolute theta power. (A) Left anterior frontal cortex (F3) interaction between conditions and moments (placebo vs. levetiracetam vs. pre vs. post) $(p=0.019)$. The paired t-test for post moment demonstrated significant difference between conditions ( $p=0.031)$; (B) midline anterior frontal cortex ( $F Z$ ) on the placebo and levetiracetam conditions ( $p=0.027)$; $(C)$ right anterior frontal cortex (F4) main effect for condition ( $P=0.000$ ); (D) main effect for pre- and post-moments was seen at F4 ( $P=0.011$ ).

neuro-enhancer. ${ }^{25,26)}$ In particular, the goal is to identify the behavioral changes produced in the reaction time, the neuropsychological changes in attention and memory, and finally examine the alleged electroencephalographic changes (qEEG) in absolute theta power in the frontal cortex. ${ }^{27,28)}$ In order to examine the effects of LEV in executive functions, a two-way-ANOVA was implemented. Specifically, two independent variables were observed: condition (placebo or LEV) and moment (pre- and post-ingestion of the LEV or placebo). This discussion has been divided into two distinct sections: i) effects of LEV on executive functions and behavioral aspects; ii) effects of LEV on electrophysiological patterns.

\section{Executive Functions (Neuropsychological Assessment)}

The neuropsychological evaluation consisted of four neuropsychological tests: Trail Making Test (A and B) (attention), Digit Span (working memory), Stroop test (inhibitory control of attention) and Tower of London (planning and decision making). ${ }^{29,30)}$ Our data indicated a change in the Trail Making Test (A), both for time and error produced by the participants. This difference was observed between placebo and LEV conditions. In particular, there was a reduction in the task execution time when the individual was under the influence of LEV. In contrast, a main effect for moment (pre and post) was found; this means that, when comparing pre- and post-moment, regardless of treatment, an error increase was observed. The Trail Making Test (A) is certainly simpler to apply than the Trail Making Test (B), since the subjects must follow a progressive numerical sequence, such as connecting points distributed randomly on an A4 sheet from a certain initial number. In our case, the subjects were always presented with the same numerical order starting with num- 

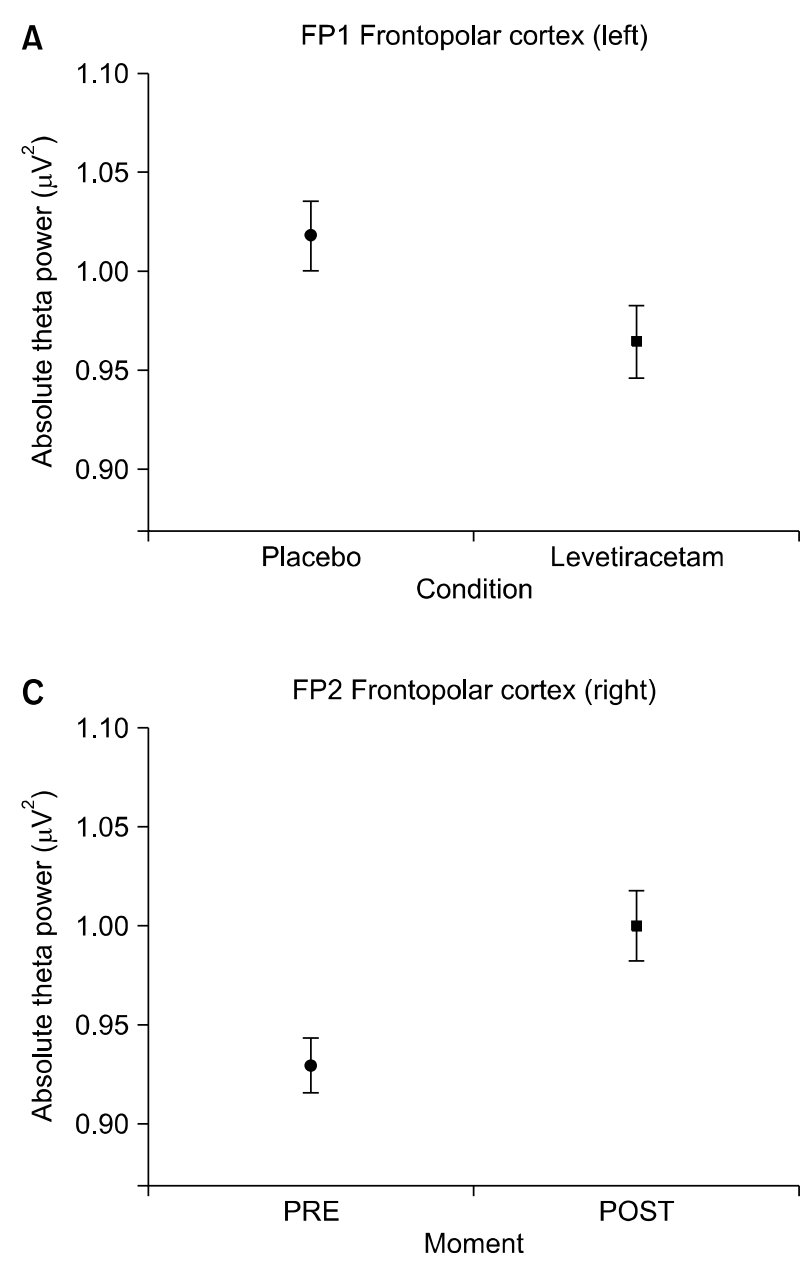

ber 1. The purpose of the Trail Making Test is to evaluate the mechanisms of attention, although there is a small attribute involving working memory (operating). ${ }^{31,32)}$ For instance, the participants need to remember the previous number during the test. Our first finding in relation to time in the Trail Making Test (A) between conditions (placebo vs. LEV) suggests that the drug had a beneficial effect on the mechanisms of attention and tracking during task execution. Particularly, this finding supports the hypothesis that tasks involving sustained attention are positively modulated by LEV. ${ }^{33-35)} \mathrm{Up}$ to now, few studies have attempted to exclude confounding effects of LEV in healthy subjects. For example, the positive effects of LEV have been associated with pathological conditions, both psychiatric and neurological or even different traumas. ${ }^{36,37)}$ Thus, our study attempts to isolate the factors that can disguise the effects of LEV alone.

Another very relevant finding in our experiment was the reduction of reaction time provided by LEV. The reaction time in a comprehensive manner, expresses the

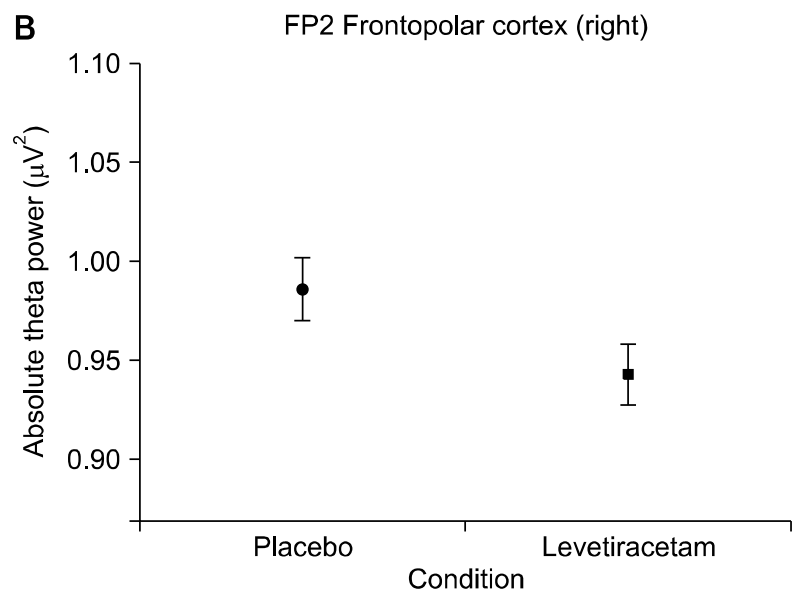

Fig. 3. Mean and standard error of absolute theta power. (A) Left frontopolar cortex (FP1) on the placebo and levetiracetam conditions ( $p=0.039)$; $(B)$ right frontopolar cortex (FP2) on the placebo and levetiracetam conditions ( $p=0.048)$; (C) main effect for pre and post moments was seen at FP2 $(p=0.001)$.

processing cycle that takes into account at least three stages in the process as a whole (uptake of the initial stimulus, choice of the best option and final motor action). Our data demonstrated a decrease of almost 15 milliseconds $(\mathrm{ms})$ in the reaction time between the LEV and placebo conditions. These data clearly indicate a modulation of LEV in the decision-making cycle in healthy individuals.

Few studies have analyzed the behavior of the reaction time and the use of LEV in healthy subjects. Studies on LEV combined with reaction time in healthy volunteers were not found. The simple reaction time was examined in an experiment with epileptic subjects and the researchers found no difference between before and after the use of LEV. ${ }^{14,38)}$ In contrast, our experiment used a reaction time of recognition between two different stimuli (e.g., square or ball), similar to the paradigm used for evoked cognitive potential (e.g., "odd-ball"). ${ }^{39-41)}$ Perhaps, the reaction time of recognition is a more sensitive measure to detect alleged changes produced by LEV, since it involves the inhibition of the motor act in non-target stimuli (e.g., ball). 
The increased complexity of the task using the reaction time of recognition makes its execution more difficult, thus, it exposes the alleged cognitive impairments produced by LEV in information processing. ${ }^{42,43)}$

\section{Effects of LEV on Electrophysiological Parameters (Absolute Theta Power)}

The electrophysiological discussion follows the same sequence of the results section. The frontal region will be discussed, starting from the most anterior electrodes until those positioned just before the central region (FP1, FP2, F7, F8, F3, Fz and F4). We recognize the complexity of the brain, in particular, the various brain regions that only belong to the cortex and its multiple communications at varying frequencies. Although this complexity is recognized, initially, we will analyze the frontal region associated with executive functions of high hierarchy, ${ }^{44-46)}$ and theta frequency by its relation to attentional processes, working memory and visuospatial aspects. ${ }^{47-49)}$ Theta activity has been associated with problem solving, several mental activities related to concentration and attentional focus. Specifically, theta is also associated with attention deficit and hyperactivity disorders. In this context, we have observed theta behavior and we will try to interpret it with the same logic applied to the alpha band. This way, theta increase could suggest a deactivation of the respective region. $^{50,51)}$

\section{Frontopolar Cortex (Anterior Prefrontal Cortex)}

The anterior region of the cerebral cortex, also known as frontopolar cortex (Brodmann area-10) has been linked to extremely complex executive functions. ${ }^{52-54)}$ The electrophysiological data for the pair of electrodes FP1 and FP2, located in the anterior prefrontal cortex region, showed a similar pattern for the main effect condition. A decrease in theta power between the placebo and LEV conditions was observed. In this case, both hemispheres had a very similar behavior. However, the FP2 electrode showed a main effect for both moment and condition, before and after the substance (placebo or LEV) ingestion. With regards to the main effect for condition (placebo vs LEV), the decrease in theta power between the placebo and LEV conditions can support the idea of a greater efficiency of the anterior cortex region. Theta power decrease suggests that the dose of LEV used $(500 \mathrm{mg})$ produced a reduction in the amount of slow frequency, compared to the placebo group. These differences could represent an improvement in some executive functions, such as: action planning, cognitive flexibility, change of plans and deci- sions, and different forms of storage. ${ }^{55,56)}$

As regards the main effect for moment (FP2), there are singularities between hemispheres. In particular, the right frontopolar cortex, as described in previous studies, participates in different cognitive processes, including prospective and working memory. ${ }^{57)}$ Prospective memory considers the idealization of future actions, i.e., remember something that will happen in the future. This increase in theta may briefly indicate a suppression of the right frontopolar hemisphere to give way to the more specialized left hemisphere in more analytical aspects. Our data suggest that the emergence of theta in the frontopolar cortex region acts as a specific inhibitory control mechanism. This inhibitory mechanism is not new; it has been seen previously in other studies ${ }^{58,59)}$ and it may partially explain our findings in relation to the reaction time. The theta power increase in the frontopolar region may contribute to the response selection, and thus produce a decrease in the time of choice and perceptual aspect, therefore generating greater efficiency in decision making. ${ }^{60,61)}$

\section{Prefrontal Inferior Gyrus (PIG): Left and Right - 47 (F7) vs. 45 (F8)}

Traditionally, functions widely associated with aspects related to language and semantics are attributed to the PIG. ${ }^{62-64)}$ This outlook highlights a localizationist approach of the neuroanatomical structures, besides not showing completely the complexity of PIG. Our results point out a quite different framework, and a supposed participation of PIG in background executive functions, which goes beyond the classical view focusing on language issues. In the PIG left region (F7), an interaction between condition and moment was observed. A detailed inspection of the interaction showed that this difference lies in the LEV condition. An increase in theta power was seen after the acute effect of LEV.

Unlike the frontopolar region (Brodmann area-10), this finding suggests that PIG responds in a manner opposite to the effects of LEV, suggesting a differentiated functionality of the left prefrontal region. The findings about F8 show a theta power decline when LEV was compared to placebo. In particular, the theta decrease clearly demonstrates an increase of activity in the right region. The right PIG is also related to operating, episodic and recognizing memory and visuospatial aspects. ${ }^{65,66)}$ Studies with depressive patients showed an increased activation in the right PIG, which was observed through functional magnetic resonance image during a recognition task. ${ }^{67,68)}$ These findings suggest that LEV can act more specifically 
on other forms of memory, and the PIG's right region can distinguish different aspects about information retrieval.

\section{Anterior Frontal Cortex (Brodmann area- 8$)-8(F 3) /$ 8L (FZ) / 8 (F4)}

The Brodmann area-8, also known as frontal visual field, plays a very important and complex role in various executive functions, ${ }^{69,70)}$ such as: working, recovery and planning memory, visual-motor attention and motor learning, proprioceptive aspects and ability to perform calculations. ${ }^{71)}$ Brodmann area-8 results were divided in different sectors: left (F3), central (FZ) and right (F4). A different behavior was detected for each of these areas. The F3 electrode showed a significant interaction between condition and moment. A careful investigation of the interaction showed a difference for the LEV condition between moments. Similarly to the behavior of the F7 electrode (left hemisphere), this region also showed a significant increase in absolute theta power after the action peak effect of LEV. This theta increase may suggest an inhibition of the left region (F7), further moving to the right region (F4). Unlike F3, the F4 electrode showed a main effect for condition, i.e., a reduction of power in the LEV condition when compared to placebo. This reduction suggests a way of neural efficiency. Such deactivation and activation may express an improvement in communication skills and functionality between hemispheres produced by LEV. This region has already been ignored in previous studies from the 1980s, ${ }^{71-73)}$ even if in different circumstances. Automatism, training and continuous repetition processes can generate this same form of efficiency of the LEV effects. ${ }^{7-78)}$ Our data also suggest the idea that there is some distinction of functions, which was evidenced by the use of LEV. According to our data, the left AFC appears to have a close relationship with inhibitory processes already described in previous studies. ${ }^{79,80)}$ This is seen through the increase in theta power after ingestion of LEV, and this inhibitory control suggests a likely mechanism for increased efficiency in this region. In contrast, the right $\mathrm{AFC}$ seems to be more associated with visuospatial and motor aspects. Maybe, the relationship between the two hemispheres may partly explain the significant difference seen in the reaction time.

This study sought to answer a single question, namely, whether LEV could be used as a cognitive amplifier in healthy subjects in a single dose. After examining the neuropsychological (attention, working memory, planning and decision making), behavioral (reaction time) and electrophysiological (absolute theta power) in the frontal re- gion, the results support the fact that LEV promotes cognitive enhancement in executive functions. In addition, our data can further investigate the control mechanisms, such as the inhibitory and excitatory mechanisms exercised by the theta frequency band. Specifically, it is possible to view and access such control mechanisms and to see how they act in different frontal cortical areas through qEEG. Although this study has shown interesting results, some limitations are listed as follows: i) we have only observed the acute effect of LEV, that is, a single dose. We haven't seen the effect of LEV on a prolonged period of time; ii) We initially limited our focus to just looking at absolute theta power, therefore leaving other variables and frequencies for future analyses: coherence, asymmetry and relative power in other cortical areas and different frequencies, such as alpha, beta and gamma are a few examples of this; iii) our neuropsychological findings are quite discrete and affirmations about the use of LEV as a cognitive enhancer should be more moderate.

\section{REFERENCES}

1. Demeter E, Sarter M. Leveraging the cortical cholinergic system to enhance attention. Neuropharmacology 2013;64: 294-304.

2. Tricco AC, Vandervaart S, Soobiah C, Lillie E, Perrier L, Chen $\mathrm{MH}$, et al. Efficacy of cognitive enhancers for Alzheimer's disease: protocol for a systematic review and network meta-analysis. Syst Rev 2012;1:31.

3. Bharadwaj PR, Bates KA, Porter T, Teimouri E, Perry G, Steele JW, et al. Latrepirdine: molecular mechanisms underlying potential therapeutic roles in Alzheimer's and other neurodegenerative diseases. Transl Psychiatry 2013;3:e332.

4. Hyun J, Baik MJ, Kang UG. Effects of psychotropic drugs on quantitative eeg among patients with schizophreniaspectrum disorders. Clin Psychopharmacol Neurosci 2011; 9:78-85.

5. Wix-Ramos R, Moreno X, Capote E, González G, Uribe E, Eblen-Zajjur A. Drug treated schizophrenia, schizoaffective and bipolar disorder patients evaluated by qEEG absolute spectral power and mean frequency analysis. Clin Psychopharmacol Neurosci 2014;12:48-53.

6. Wood S, Sage JR, Shuman T, Anagnostaras SG. Psychostimulants and cognition: a continuum of behavioral and cognitive activation. Pharmacol Rev 2013;66:193-221.

7. Sadasivan S, Pond BB, Pani AK, Qu C, Jiao Y, Smeyne RJ. Methylphenidate exposure induces dopamine neuron loss and activation of microglia in the basal ganglia of mice. PLoS One 2012;7:e33693.

8. Stip E, Chouinard S, Boulay LJ. On the trail of a cognitive enhancer for the treatment of schizophrenia. Prog Neuropsychopharmacol Biol Psychiatry 2005;29:219-232.

9. Weeks AM, Harms JE, Partin KM, Benveniste M. Functional insight into development of positive allosteric modulators of AMPA receptors. Neuropharmacology 2014; 85:57-66.

10. Lynch G, Cox CD, Gall CM. Pharmacological enhancement of memory or cognition in normal subjects. Front Syst 
Neurosci 2014;8:90.

11. Bakker A, Krauss GL, Albert MS, Speck CL, Jones LR, Stark CE, et al. Reduction of hippocampal hyperactivity improves cognition in amnestic mild cognitive impairment. Neuron 2012;74:467-474.

12. Stockis A, Sargentini-Maier ML, Otoul C, Connor A, Wilding I, Wray H. Assessment of levetiracetam bioavailability from targeted sites in the human intestine using remotely activated capsules and gamma scintigraphy: Open-label, single-dose, randomized, four-way crossover study in healthy male volunteers. Clin Ther 2010;32:18131821.

13. Mittapalli GK, Roberts E. Structure activity relationships of novel antiepileptic drugs. Curr Med Chem 2014;21:722-754.

14. Lin CC, Ju MS, Chen CW, Hwang JC, Tsai JJ. Effects of levetiracetam on $\mu$ rhythm in persons with epilepsy. J Clin Neurosci 2013;20:686-691.

15. Başar E, Güntekin B. A short review of alpha activity in cognitive processes and in cognitive impairment. Int $J$ Psychophysiol 2012;86:25-38.

16. Farr OM, Zhang S, Hu S, Matuskey D, Abdelghany O, Malison RT, et al. The effects of methylphenidate on resting-state striatal, thalamic and global functional connectivity in healthy adults. Int $J$ Neuropsychopharmacol 2014; 17:1177-1191.

17. Jensen O, Bonnefond M. Prefrontal $\alpha$ - and $\beta$-band oscillations are involved in rule selection. Trends Cogn Sci 2013;17:10-12.

18. Jasper $\mathrm{H}$. The ten-twenty electrode system of the International Federation. Electroencephalogr Clin Neurophysiol 1958;10: 371-375.

19. Perfetti B, Moisello C, Lanzafame S, Varanese S, Landsness $\mathrm{EC}$, Onofrj M, et al. Attention modulation regulates both motor and non-motor performance: a high-density EEG study in Parkinson's disease. Arch Ital Biol 2010;148:279288.

20. Davis CE, Hauf JD, Wu DQ, Everhart DE. Brain function with complex decision making using electroencephalography. Int J Psychophysiol 2011;79:175-183.

21. Davydov DM, Polunina AG. Heroin abusers' performance on the Tower of London Test relates to the baseline EEG alpha2 mean frequency shifts. Prog Neuropsychopharmacol Biol Psychiatry 2004;28:1143-1152.

22. Helmstaedter C, Witt JA. Cognitive outcome of antiepileptic treatment with levetiracetam versus carbamazepine monotherapy: a non-interventional surveillance trial. Epilepsy Behav 2010;18:74-80.

23. Park SP, Kwon OY. Increased EEG current-source density in the high Beta frequency band induced by levetiracetam adjunctive therapy in refractory partial epilepsy. J Clin Neurol 2009;5:178-185.

24. Smith ME, McEvoy LK, Gevins A. Neurophysiological indices of strategy development and skill acquisition. Brain Res Cogn Brain Res 1999;7:389-404.

25. Sanchez PE, Zhu L, Verret L, Vossel KA, Orr AG, Cirrito $\mathrm{JR}$, et al. Levetiracetam suppresses neuronal network dysfunction and reverses synaptic and cognitive deficits in an Alzheimer's disease model. Proc Natl Acad Sci U S A 2012; 109:E2895-E2903.

26. Cho JR, Koo DL, Joo EY, Yoon SM, Ju E, Lee J, et al. Effect of levetiracetam monotherapy on background EEG activity and cognition in drug-naïve epilepsy patients. Clin Neurophysiol 2012;123:883-891.

27. Putman P, Verkuil B, Arias-Garcia E, Pantazi I, van Schie C. EEG theta/beta ratio as a potential biomarker for atten- tional control and resilience against deleterious effects of stress on attention. Cogn Affect Behav Neurosci 2014;14: 782-791.

28. Roux F, Uhlhaas PJ. Working memory and neural oscillations: $\alpha-\gamma$ versus $\theta-\gamma$ codes for distinct $W M$ information? Trends Cogn Sci 2014;18:16-25.

29. Perumparaichallai RK, Husk KL, Myles SM, Klonoff PS. The relationship of neuropsychological variables to driving status following holistic neurorehabilitation. Front Neurol 2014;5:56.

30. Yao X, Yu Q, Yang E, Ouyang H, Chen Y, Yang W, et al. Executive dysfunction in patients with temporal lobe epilepsy and its correlation with P300. Zhonghua Yi Xue Za Zhi 2014;94:521-524.

31. Nakahachi T, Ishii R, Iwase M, Canuet L, Takahashi H, Kurimoto R, et al. Frontal cortex activation associated with speeded processing of visuospatial working memory revealed by multichannel near-infrared spectroscopy during Advanced Trail Making Test performance. Behav Brain Res 2010;215:21-27.

32. Seo J, Kim YT, Song HJ, Lee HJ, Lee J, Jung TD, et al. Stronger activation and deactivation in archery experts for differential cognitive strategy in visuospatial working memory processing. Behav Brain Res 2012;229:185-193.

33. Koo DL, Hwang KJ, Kim D, Kim YJ, Kim JY, Shin W, et al. Effects of levetiracetam monotherapy on the cognitive function of epilepsy patients. Eur Neurol 2013;70:88-94.

34. Consoli D, Bosco D, Postorino P, Galati F, Plastino M, Perticoni GF, et al; EPIC Study. Levetiracetam versus carbamazepine in patients with late poststroke seizures: a multicenter prospective randomized open-label study (EpIC Project). Cerebrovasc Dis 2012;34:282-289.

35. Cumbo E, Ligori LD. Levetiracetam, lamotrigine, and phenobarbital in patients with epileptic seizures and Alzheimer's disease. Epilepsy Behav 2010;17:461-466.

36. Mbizvo GK, Dixon P, Hutton JL, Marson AG. The adverse effects profile of levetiracetam in epilepsy: a more detailed look. Int J Neurosci 2014;124:627-634.

37. Hagemann A, May TW, Nieder E, Witte-Bölt K, PohlmannEden B, Elger CE, et al. Quality of life, anxiety and depression in adult patients after add-on of levetiracetam and conversion to levetiracetam monotherapy. Epilepsy Res 2013; 104:140-150.

38. Mecarelli O, Vicenzini E, Pulitano P, Vanacore N, Romolo FS, Di Piero V, et al. Clinical, cognitive, and neurophysiologic correlates of short-term treatment with carbamazepine, oxcarbazepine, and levetiracetam in healthy volunteers. Ann Pharmacother 2004;38:1816-1822.

39. Saenghong N, Wattanathorn J, Muchimapura S, Tongun T, Piyavhatkul N, Banchonglikitkul C, et al. Zingiber officinale improves cognitive function of the middle-aged healthy women. Evid Based Complement Alternat Med 2012;2012: 383062.

40. Zhang Y, Lehmann M, Shobeiry A, Höfer D, Johannes S, Emrich $\mathrm{HM}$, et al. Effects of quetiapine on cognitive functions in schizophrenic patients: a preliminary single-trial ERP analysis. Pharmacopsychiatry 2009;42:129-134.

41. Osinsky R, Walter H, Hewig J. What is and what could have been: an ERP study on counterfactual comparisons. Psychophysiology 2014;51:773-781.

42. Higgins GA, Breysse N, Undzys E, Derksen DR, Jeffrey M, Scott BW, et al. Comparative study of five antiepileptic drugs on a translational cognitive measure in the rat: relationship to antiepileptic property. Psychopharmacology (Berl) 2010;207:513-527. 
43. Shannon HE, Love PL. Effects of antiepileptic drugs on attention as assessed by a five-choice serial reaction time task in rats. Epilepsy Behav 2005;7:620-628.

44. Morein-Zamir S, Dodds C, van Hartevelt TJ, Schwarzkopf $\mathrm{W}$, Sahakian B, Müller U, et al. Hypoactivation in right inferior frontal cortex is specifically associated with motor response inhibition in adult ADHD. Hum Brain Mapp 2014; 35:5141-5152.

45. Cardoso Cde O, Branco LD, Cotrena C, Kristensen CH, Schneider Bakos DD, Fonseca RP. The impact of frontal and cerebellar lesions on decision making: evidence from the Iowa Gambling Task. Front Neurosci 2014;8:61.

46. Gonzalez CL, Mills KJ, Genee I, Li F, Piquette N, Rosen $\mathrm{N}$, et al. Getting the right grasp on executive function. Front Psychol 2014;5:285.

47. Griesmayr B, Berger B, Stelzig-Schoeler R, Aichhorn W, Bergmann J, Sauseng P. EEG theta phase coupling during executive control of visual working memory investigated in individuals with schizophrenia and in healthy controls. Cogn Affect Behav Neurosci 2014;14:1340-1355.

48. Wang JR, Hsieh S. Neurofeedback training improves attention and working memory performance. Clin Neurophysiol 2013;124:2406-2420.

49. Polanía R, Nitsche MA, Korman C, Batsikadze G, Paulus $\mathrm{W}$. The importance of timing in segregated theta phase-coupling for cognitive performance. Curr Biol 2012;22:13141318.

50. Brazier MA, Casby JU. Cross-correlation and autocorrelation studies of electroencephalographic potentials. Electroencephalogr Clin Neurophysiol 1952;4:201-211.

51. Buyck I, Wiersema JR. Task-related electroencephalographic deviances in adults with attention deficit hyperactivity disorder. Neuropsychology 2014. In Press.

52. Barban F, Carlesimo GA, Macaluso E, Caltagirone C, Costa A. Functional interplay between stimulus-oriented and stimulus-independent attending during a prospective memory task. Neuropsychologia 2014;53:203-212.

53. Dalwani MS, Tregellas JR, Andrews-Hanna JR, MikulichGilbertson SK, Raymond KM, Banich MT, et al. Default mode network activity in male adolescents with conduct and substance use disorder. Drug Alcohol Depend 2014;134: 242-250.

54. Chiang TC, Liang $\mathrm{KC}$, Chen $\mathrm{JH}$, Hsieh $\mathrm{CH}$, Huang YA. Brain deactivation in the outperformance in bimodal tasks: an FMRI study. PLoS One 2013;8:e77408.

55. Devi L, Ohno M. Effects of levetiracetam, an antiepileptic drug, on memory impairments associated with aging and Alzheimer's disease in mice. Neurobiol Learn Mem 2013; 102:7-11.

56. Taylor S, Heinrichs RJ, Janzen JM, Ehtisham A. Levetiracetam is associated with improved cognitive outcome for patients with intracranial hemorrhage. Neurocrit Care 2011;15:8084.

57. Costa A, Oliveri M, Barban F, Bonnì S, Koch G, Caltagirone $\mathrm{C}$, et al. The right frontopolar cortex is involved in visual-spatial prospective memory. PLoS One 2013;8: e56039.

58. Schmajuk M, Liotti M, Busse L, Woldorff MG. Electrophysiological activity underlying inhibitory control processes in normal adults. Neuropsychologia 2006;44:384-395.

59. Rubia K, Smith AB, Brammer MJ, Taylor E. Right inferior prefrontal cortex mediates response inhibition while mesial prefrontal cortex is responsible for error detection. Neuroimage 2003;20:351-358

60. Guitart-Masip M, Barnes GR, Horner A, Bauer M, Dolan
RJ, Duzel E. Synchronization of medial temporal lobe and prefrontal rhythms in human decision making. $J$ Neurosci 2013;33:442-451.

61. Remondes M, Wilson MA. Cingulate-hippocampus coherence and trajectory coding in a sequential choice task. Neuron 2013;80:1277-1289.

62. Belyk M, Brown S. Perception of affective and linguistic prosody: an ALE meta-analysis of neuroimaging studies. Soc Cogn Affect Neurosci 2014;9:1395-1403.

63. Zhuang J, Tyler LK, Randall B, Stamatakis EA, MarslenWilson WD. Optimally efficient neural systems for processing spoken language. Cereb Cortex 2014;24:908-918.

64. Fridriksson J, Hubbard HI, Hudspeth SG, Holland AL, Bonilha L, Fromm D, et al. Speech entrainment enables patients with Broca's aphasia to produce fluent speech. Brain 2012;135:3815-3829.

65. Hanslmayr S, Matuschek J, Fellner MC. Entrainment of prefrontal beta oscillations induces an endogenous echo and impairs memory formation. Curr Biol 2014;24:904-909.

66. Vartanian O, Bouak F, Caldwell JL, Cheung B, Cupchik G, Jobidon ME, et al. The effects of a single night of sleep deprivation on fluency and prefrontal cortex function during divergent thinking. Front Hum Neurosci 2014;8:214.

67. Dietsche B, Backes H, Stratmann M, Konrad C, Kircher T, Krug A. Altered neural function during episodic memory encoding and retrieval in major depression. Hum Brain Mapp 2014;35:4293-4302.

68. Thompson DG, Kesler SR, Sudheimer K, Mehta KM, Thompson LW, Marquett RM, et al. FMRI activation during executive function predicts response to cognitive behavioral therapy in older, depressed adults. Am J Geriatr Psychiatry 2015;23:13-22.

69. Lanzilotto M, Perciavalle V, Lucchetti C. A new field in monkey's frontal cortex: premotor ear-eye field (PEEF). Neurosci Biobehav Rev 2013;37:1434-1444.

70. Kerestes R, Ladouceur CD, Meda S, Nathan PJ, Blumberg $\mathrm{HP}$, Maloney K, et al. Abnormal prefrontal activity subserving attentional control of emotion in remitted depressed patients during a working memory task with emotional distracters. Psychol Med 2012;42:29-40.

71. Brooks JO 3rd, Bearden CE, Hoblyn JC, Woodard SA, Ketter TA. Prefrontal and paralimbic metabolic dysregulation related to sustained attention in euthymic older adults with bipolar disorder. Bipolar Disord 2010;12:866-874.

72. Hatfield FM, Patterson KE. Interpretation of spelling disorders in aphasia: impact of recent developments in cognitive psychology. Adv Neurol 1984;42:183-192.

73. Frenkel-Toledo S, Bentin S, Perry A, Liebermann DG, Soroker N. Dynamics of the EEG power in the frequency and spatial domains during observation and execution of manual movements. Brain Res 2013;1509:43-57.

74. van Driel J, Ridderinkhof KR, Cohen MX. Not all errors are alike: theta and alpha EEG dynamics relate to differences in error-processing dynamics. J Neurosci 2012;32: 16795-16806.

75. van Leeuwen S, Singer W, Melloni L. Meditation increases the depth of information processing and improves the allocation of attention in space. Front Hum Neurosci 2012;6: 133.

76. Christova M, Rafolt D, Golaszewski S, Nardone R, Gallasch E. Electrical stimulation during skill training with a therapeutic glove enhances the induction of cortical plasticity and has a positive effect on motor memory. Behav Brain Res 2014;270:171-178.

77. Engen H, Kanske P. How working memory training im- 
proves emotion regulation: neural efficiency, effort, and transfer effects. J Neurosci 2013;33:12152-12153.

78. Provost A, Johnson B, Karayanidis F, Brown SD, Heathcote A. Two routes to expertise in mental rotation. Cogn Sci 2013;37:1321-1342.

79. Erika-Florence M, Leech R, Hampshire A. A functional net- work perspective on response inhibition and attentional control. Nat Commun 2014;5:4073.

80. Crozier S, Sirigu A, Lehéricy S, van de Moortele PF, Pillon $\mathrm{B}$, Grafman J, et al. Distinct prefrontal activations in processing sequence at the sentence and script level: an fMRI study. Neuropsychologia 1999;37:1469-1476. 